\title{
ON THE COMPUTATION OF COEFFICIENTS OF MODULAR FORMS: THE REDUCTION MODULO $p$ APPROACH
}

\author{
JINXIANG ZENG AND LINSHENG YIN
}

\begin{abstract}
In this paper, we present a probabilistic algorithm to compute the coefficients of modular forms of level one. Focusing on Ramanujan's tau function, we give the explicit complexity of the algorithm. From a practical viewpoint, the algorithm is particularly well suited for implementations.
\end{abstract}

\section{INTRODUCTION AND MAIN RESULTS}

In the book 13, Couveignes, Edixhoven et al. described an algorithm for computing coefficients of modular forms for the group $\mathrm{SL}_{2}(\mathbb{Z})$, and Bruin $[7$ generalized the method to modular forms for the congruence subgroups of the form $\Gamma_{1}(n)$. Their methods lead to polynomial time algorithms for computing coefficients of modular forms. However, efficient ways to implement the algorithms and explicit complexity analysis are still being studied. Working with the complex number field, Bosman's explicit computations show the power of these new methods; see [13. As one of the applications, he largely improved the known result on Lehmer's nonvanishing conjecture for Ramanujan's tau function. For the recent progress in this direction, see [25]. Following Couveignes's idea [9], we give a probabilistic algorithm, which seems to be more suitable to deal with complexity analysis. Instead of using BrillNoether's algorithm, we work with the function field of the modular curve, using Heß's algorithm to make computations in the Jacobian of modular curves.

We illustrate our method on the discriminant modular form, which is defined as

$$
\Delta(q)=q \prod_{n=1}^{\infty}\left(1-q^{n}\right)^{24}=\sum_{n=1}^{\infty} \tau(n) q^{n},
$$

where $z \in \mathcal{H}, q=e^{2 \pi i z}$.

Let $p$ be a prime; using Deligne's bound we have $|\tau(p)| \leq 2 p^{11 / 2}$. Therefore to compute $\tau(p)$ it suffices to compute $\tau(p) \bmod \ell$ for all primes $\ell$ bounded above by a constant in $\mathrm{O}(\log p)$. Let $\ell$ be a prime; the $\bmod -\ell$ Galois representation associated to $\Delta(q)$ is denoted as

$$
\rho_{\ell}: \operatorname{Gal}(\overline{\mathbb{Q}} / \mathbb{Q}) \rightarrow \mathrm{GL}_{2}\left(\mathbb{F}_{\ell}\right),
$$

which satisfies that for any prime $p \neq \ell$, the characteristic polynomial of the Frobenius endomorphism Frob $_{p}$ is $x^{2}-\tau(p) x+p^{11} \bmod \ell$. Therefore, to compute $\tau(p)$ $\bmod \ell$ it suffices to compute the Galois representation $\rho_{\ell}$. It is well known that

Received by the editor May 17, 2013 and, in revised form, September 17, 2013.

2010 Mathematics Subject Classification. Primary 11F30, 11G20, 11Y16, 14Q05, 14 H05.

Key words and phrases. Modular forms, Hecke algebra, modular curves, elliptic curves, Jacobian.

This work was partially supported by NSFC grant No.11271212. 
$\rho_{\ell}$ can be realized by the subspace $V_{\ell}$ in the $\ell$-torsions $J_{1}(\ell)(\overline{\mathbb{Q}})[\ell]$ of the Jacobian variety $J_{1}(\ell)$ of the modular curve $X_{1}(\ell)$, which can be written as a finite intersection

$$
V_{\ell}=\bigcap_{1 \leq i \leq\left(\ell^{2}-1\right) / 6} \operatorname{ker}\left(T_{i}-\tau(i), J_{1}(\ell)(\overline{\mathbb{Q}})[\ell]\right),
$$

where $T_{i}, i=1, \ldots, \frac{\ell^{2}-1}{6}$ are Hecke operators. Indeed $V_{\ell}$ is a group scheme over $\mathbb{Q}$ of order $\ell^{2}$, which is called the Ramanujan subspace [9]. As showed in [13, the heights of the elements of $V_{\ell}$ are well bounded, which enables us to know $V_{\ell}$ explicitly. More precisely, we have function $\iota: V_{\ell} \rightarrow \mathbb{A}_{\mathbb{Q}}^{1}$, such that the heights of the coefficients of $P(X):=\prod_{\alpha \in V_{\ell} \backslash \mathrm{O}}(X-\iota(\alpha))$ are bounded above by $\mathrm{O}\left(\ell^{\delta}\right)$, where the constant $\delta$ is independent of $\ell$ and the function $\iota$ is constructed explicitly in [7].

Our approach is to compute $V_{\ell} \bmod p$ for sufficiently many auxiliary small primes $p$ as in Schoof's algorithm, and then reconstruct $V_{\ell}$ by the Chinese Remainder Theorem. The main results of the paper are as follows.

Theorem 1.1. Let $\ell \geq 13$ be a prime and $p$ an s-good prime. Given the Zeta function of the modular curve $X_{1}(\ell)_{\mathbb{F}_{p}}$, then $V_{\ell} \bmod p$ can be computed in time $O\left(\ell^{4+2 \omega+\epsilon} \cdot \log ^{1+\epsilon} p \cdot(\ell+\log p)\right)$.

\section{Corollary 1.2.}

(1) The Ramanujan subspace $V_{\ell}$ can be computed in time $O\left(\ell^{6+2 \omega+\delta+\epsilon}\right)$.

(2) For prime $p, \tau(p)$ can be computed in time $O\left(\log ^{7+2 \omega+\delta+\epsilon} p\right)$.

Remark 1.3. See Definition 4.2 for the definition of $s$-good prime. The constant $\omega$ refers to the fact that the complexity of a single group operation in the Jacobian variety $J_{1}(\ell)$ is in $\mathrm{O}\left(g^{\omega}\right)$, where $g$ is the genus of the modular curve $X_{1}(\ell)$. Using Khuri-Makdisi's algorithm, the constant $\omega$ can be 2.376. Using Heß's algorithm, $\omega$ is known to be in $[2,4]$. The constant $\delta$ is bigger than $\operatorname{dim} V_{\ell}=2$.

Theorem 1.4. The nonvanishing of $\tau(n)$ holds for all $n$ such that

$$
n<982149821766199295999 \approx 9 \cdot 10^{20} .
$$

Remark 1.5. In [5] the nonvanishing of $\tau(n)$ was verified for all $n$ such that

$$
n<22798241520242687999 \approx 2 \cdot 10^{19} .
$$

Notation. The running time will always be measured in bit operations. Using FFT, multiplication of two $n$-bit length integers can be done in $\mathrm{O}\left(n^{1+\epsilon}\right)$ time. Multiplication in finite field $\mathbb{F}_{q}$ can be done in $\mathrm{O}\left(\log ^{1+\epsilon} q\right)$.

The paper is organized as follows. In Section 2 we provide some necessary background on computing a convenient plane model and the function field of the modular curve; results for computing isogenies of an elliptic curve over finite fields are also recalled. A better bound on the generators of the maximal ideal of Hecke algebra is proved, which is used to reduce the complexity of the algorithm.

Section 3 contains the application of Heß's algorithm to the computation in the Jacobian of the modular curve over finite fields. Here, we introduce methods to find the correspondence between the places of the function field and the cusps of the modular curve, and compute the action of Hecke operators on places of the function field.

The main algorithm is given in Section 4, including complexity analysis.

Section 5 is concerned with some real computations of Ramanujan's tau function. All of our computations are based on Magma computational algebra system [4. 


\section{FunCtion Field of MOdUlar CURVES}

In this section we study the plane model and the function field of the modular curve $X_{1}(\ell)$.

Let $\Gamma_{1}(\ell)$ be a congruence subgroup of $\mathrm{SL}_{2}(\mathbb{Z})$, defined as

$$
\Gamma_{1}(\ell)=\left\{\left[\begin{array}{ll}
a & b \\
c & d
\end{array}\right] \in \mathrm{SL}_{2}(\mathbb{Z}):\left[\begin{array}{ll}
a & b \\
c & d
\end{array}\right] \equiv\left[\begin{array}{ll}
1 & * \\
0 & 1
\end{array}\right](\bmod \ell)\right\}
$$

(where "*" means "unspecified"), and $\mathcal{H}$ the upper half complex plane. The modular curve $Y_{1}(\ell)$ is defined as the quotient space of orbits under $\Gamma_{1}(\ell)$,

$$
Y_{1}(\ell)=\Gamma_{1}(\ell) \backslash \mathcal{H}
$$

We can add cusps $\mathbb{P}^{1}(\mathbb{Q})$ to $Y_{1}(\ell)$ to compactify it and obtain the modular curve

$$
X_{1}(\ell)=\Gamma_{1}(\ell) \backslash \mathcal{H} \cup \mathbb{P}^{1}(\mathbb{Q}) .
$$

This complex algebraic curve is defined over $\mathbb{Q}$, denoted by $X_{1}(\ell)_{\mathbb{Q}}$. Moreover, for $\ell \geq 5, X_{1}(\ell)$ has a natural model over $\mathbb{Z}[1 / \ell]$. Let $K$ be a number field. Then $K$-valued points of $Y_{1}(\ell)_{\mathbb{Q}}$ can be interpreted as

$$
Y_{1}(\ell)_{\mathbb{Q}}(K)=\{(E, P): E / K, P \in E[\ell](K) \backslash O\} / \sim,
$$

where $E$ is an elliptic curve over $K, P$ is a $K$-rational point of order $\ell$, and $\left(E_{1}, P_{1}\right) \sim\left(E_{2}, P_{2}\right)$ means that there exists an $\bar{K}$-isomorphism $\phi: E_{1} \rightarrow E_{2}$, such that $\phi\left(P_{1}\right)=P_{2}$. Such a moduli interpretation implies a way to obtain a plane model of the modular curve, as in the following proposition (see [3]).

Proposition 2.1. Suppose that $\ell \geq 4$. Then every $K$-isomorphism class of pairs $(E, P)$ with $E$ an elliptic curve over $K$ and $P \in E(K)$ a torsion point of order $\ell$ contains a unique model of the Tate normal form

$$
E_{(b, c)}: y^{2}+(1-c) x y-b y=x^{3}-b x^{2}, P=(0,0),
$$

with $c \in K, b \in K^{*}$.

Thus, points of $X_{1}(\ell)$ can be represented as pairs $(b, c)$ in a unique way. The $\ell$-th division polynomial gives a polynomial in $b$ and $c$, which defines a plane curve that is birationally equivalent to $X_{1}(\ell)$. The defining equation becomes much simpler, through a carefully chosen sequence of rational transformations. We use the table listed in 30; for example, a plane model of $X_{1}(19)$ is

$$
\begin{aligned}
f(x, y)= & y^{5}-\left(x^{2}+2\right) y^{4}-\left(2 x^{3}+2 x^{2}+2 x-1\right) y^{3}+\left(x^{5}+3 x^{4}+7 x^{3}+6 x^{2}+2 x\right) y^{2} \\
& -\left(x^{5}+2 x^{4}+4 x^{3}+3 x^{2}\right) y+x^{3}+x^{2}
\end{aligned}
$$

where

$$
r=1+\frac{x(x+y)(y-1)}{(x+1)\left(x^{2}-x y+2 x-y^{2}+y\right)}, s=1+\frac{x(y-1)}{(x+1)(x-y+1)},
$$

and

$$
c=s(r-1), b=r c .
$$

So the function field of $X_{1}(19)$ over $\mathbb{Q}$ is

$$
\mathbb{Q}\left(X_{1}(19)\right)=\mathbb{Q}(b, c)=\mathbb{Q}(x)[y] /(f(x, y)) .
$$


Cusps of $X_{1}(\ell)$ correspond to those pairs $(b, c)$ such that the $j$-invariant $j\left(E_{(b, c)}\right)$ $=\infty$. For $\ell$ an odd prime, the modular curve $X_{1}(\ell)$ has $\ell-1$ cusps, half of which are in $X_{1}(\ell)(\mathbb{Q})$ and the rest are defined over the maximal real subfield of $\mathbb{Q}\left(\zeta_{\ell}\right)$. Accordingly, every $\mathbb{Q}$-rational cusp corresponds to a degree one place of $\mathbb{Q}\left(X_{1}(\ell)\right)$ and the $\mathbb{Q}\left(\zeta_{\ell}\right)$-cusps corresponds to a degree $\frac{\ell-1}{2}$ place of $\mathbb{Q}\left(X_{1}(\ell)\right)$. Let $O_{1}, \ldots, O_{(\ell-1) / 2}$ be the $\mathbb{Q}$-rational cusps; it is easy to get these places after writing the exact expression of $j\left(E_{(b, c)}\right)$ in variables $x, y$. As in the above example, one of the $\mathbb{Q}$-rational cusps looks like

$$
\begin{aligned}
O_{1}=\left(x, \frac{y^{4}}{x^{4}+x^{3}}+\frac{y^{3}\left(-x^{3}-x^{2}+x-1\right)}{x^{4}+x^{3}}\right. & \\
& \left.+\frac{y^{2}\left(-x^{3}-2 x^{2}-2 x-2\right)}{x^{3}+x^{2}}+\frac{2 y}{x}+\frac{2 x-1}{x}\right),
\end{aligned}
$$

where the place $O_{1}$ is represented by a prime ideal of the maximal orders of the function field $\mathbb{Q}\left(X_{1}(\ell)\right)$.

It's well known that the modular curve $X_{1}(\ell)$ has good reduction at prime $p \nmid \ell$; see [11. The reduction curve is denoted by $X_{1}(\ell)_{\mathbb{F}_{p}}$. Having a nonsingular affine model of $X_{1}(\ell)$, we can easily get an affine model for $X_{1}(\ell)_{\mathbb{F}_{p}}$ and then have the function field of $X_{1}(\ell)_{\mathbb{F}_{p}}$. For simplicity, the plane model of $X_{1}(\ell)_{\mathbb{F}_{p}}$ and the $\mathbb{F}_{p^{-}}$ rational cusps of $X_{1}(\ell)_{\mathbb{F}_{p}}$, which are the reductions of the $\mathbb{Q}$-rational cusps of $X_{1}(\ell)$, are also denoted by $f(x, y)$ and $O_{i}, i \in\left\{1, \ldots, \frac{\ell-1}{2}\right\}$, respectively.

The Ramanujan subspace $V_{\ell} \bmod p$ is a subgroup scheme of the Jacobian variety $J_{1}(\ell)_{\mathbb{F}_{p}}$ of $X_{1}(\ell)_{\mathbb{F}_{p}}$. Similarly, it can be written as a finite intersection

$$
V_{\ell} \bmod p=\bigcap_{1 \leq i \leq \frac{\ell^{2}-1}{6}} \operatorname{ker}\left(T_{i}-\tau(i), J_{1}(\ell)_{\mathbb{F}_{p}}[\ell]\right),
$$

where $T_{i}, 1 \leq i \leq \frac{\ell^{2}-1}{6}$, are Hecke operators, and the number $\frac{\ell^{2}-1}{6}$ follows from [29]. In fact, the Hecke algebra $\mathbb{T}=\mathbb{Z}\left[T_{n}: n \geq 1\right] \subset \operatorname{End}\left(J_{1}(\ell)\right)$ is a free $\mathbb{Z}$-module of rank $g=\frac{(\ell-5)(\ell-7)}{24}$. After representing each Hecke operator as a matrix, see [13], a set of generators for $\mathbb{T}$ can be extracted from $T_{1}, \ldots, T_{\left(\ell^{2}-1\right) / 6}$ by solving linear equations. For example, when the level $\ell=17$, the Hecke algebra $\mathbb{T}$ is equal to $\mathbb{Z} T_{1}+\ldots+\mathbb{Z} T_{48}$ as an $\mathbb{Z}$-module, which can be replaced by $\mathbb{Z} T_{1}+\mathbb{Z} T_{2}+\mathbb{Z} T_{3}+\mathbb{Z} T_{4}+\mathbb{Z} T_{6}$ as a free $\mathbb{Z}$-module, so there are fewer Hecke operators and isogenies of lower degrees needed to take into account. But, in practice, we can do much better; notice that our goal is to find nonzero elements in $J_{1}(\ell)_{\mathbb{F}_{p}}[\ell]$, which are annihilated by $T_{k}-\tau(k), k \geq 1$. Assume there is an element $D \in J_{1}(\ell)_{\mathbb{F}_{p}}[\ell]$ satisfying $\left(T_{2}-\tau(2)\right)(D)=0$, and $T_{k}-\tau(k)$ is divisible by $T_{2}-\tau(2)$ in $\mathbb{T} \otimes \mathbb{F}_{\ell}$ for all $k \geq 2$. Then $\left(T_{k}-\tau(k)\right)(D), k \geq 2$, is equal to zero automatically, which implies that $D \in V_{\ell} \bmod p$. We can check that when the level $\ell \in\{13,17,19,23,29,37,41,43\}$, for any $k \geq 2, T_{k}-\tau(k)$ is divisible by $T_{2}-\tau(2)$ in $\mathbb{T} \otimes \mathbb{F}_{\ell}$, while $\ell=31, T_{3}-\tau(3)$ satisfies this property. In view of these observations, we proved the following proposition.

Proposition 2.2. Let $\ell \geq 13$ be a prime and $S_{2}\left(\Gamma_{1}(\ell)\right)$ be the space of cusp modular forms of weight 2 level $\ell$ over $\mathbb{C}$. Let $\mathbb{T}=\mathbb{Z}\left[T_{n}: n \geq 1\right] \subset \operatorname{End}\left(S_{2}\left(\Gamma_{1}(\ell)\right)\right)$ be the Hecke algebra and $\mathfrak{m}$ the maximal ideal generated by $\ell$ and the $T_{n}-\tau(n)$ with $n \geq 1$. Then $\mathfrak{m}$ can be generated by $\ell$ and the $T_{n}-\tau(n)$ with $1 \leq n \leq \ell+2$. 
Proof. Let $R:=\mathbb{T} \otimes \mathbb{F}_{\ell}$. Then $R$ is an Artin ring, which can be decomposed as

$$
R=\prod_{\wp} R_{\wp},
$$

where $\wp$ runs through all maximal ideals of $R$ and $R_{\wp}$ is the localization of $R$ at $\wp$.

Let $\tilde{\mathfrak{m}}$ be the image of $\mathfrak{m}$ in $R$. Then, it is enough to prove $\tilde{\mathfrak{m}}$ can be generated by the $T_{n}-\tau(n)$ with $1 \leq n \leq \ell+2$, which is equivalent to showing that any $T_{k}-\tau(k), k>\ell+2$, can be represented as

$$
T_{k}-\tau(k)=\sum_{i=1}^{\ell+2} A_{i} \cdot\left(T_{i}-\tau(i)\right),
$$

where $A_{i}$ are operators in $R$.

Let $S_{2}\left(\Gamma_{1}(\ell) ; \overline{\mathbb{F}}_{\ell}\right):=S_{2}\left(\Gamma_{1}(\ell) ; \mathbb{Z}\right) \otimes \overline{\mathbb{F}}_{\ell}$, which is an $R$-module, decomposed as

$$
S_{2}\left(\Gamma_{1}(\ell) ; \overline{\mathbb{F}}_{\ell}\right)=\prod_{\wp} S_{2}\left(\Gamma_{1}(\ell) ; \overline{\mathbb{F}}_{\ell}\right)_{\wp},
$$

where $\wp$ runs through all maximal ideals of $R$. Here $S_{2}\left(\Gamma_{1}(\ell) ; \overline{\mathbb{F}}_{\ell}\right)_{\wp}$ is the localization of $S_{2}\left(\Gamma_{1}(\ell) ; \overline{\mathbb{F}}_{\ell}\right)$ at $\wp$, which is an $R_{\wp}$-module. To show (2.5), it's enough to show that for each $\wp$, the action $T_{k}-\tau(k), k>\ell+2$, on $S_{2}\left(\Gamma_{1}(\ell) ; \overline{\mathbb{F}}_{\ell}\right)_{\wp}$ can be represented as

$$
T_{k}-\tau(k)=\sum_{i=1}^{\ell+2} B_{i} \cdot\left(T_{i}-\tau(i)\right),
$$

where $B_{i}$ are operators in $R_{\wp}$.

The maximal ideal $\wp$ of $R$ corresponds to a $\operatorname{Gal}\left(\overline{\mathbb{F}}_{\ell} / \mathbb{F}_{\ell}\right)$-conjugate class $[f]$ of normalized eigenforms in $S_{2}\left(\Gamma_{1}(\ell) ; \overline{\mathbb{F}}_{\ell}\right)$; they are newforms of level $\ell$. Thus the localization $S_{2}\left(\Gamma_{1}(\ell) ; \overline{\mathbb{F}}_{\ell}\right)_{\wp}$ is a vector space spanned by these newforms, so $R_{\wp}$ is a field isomorphic to the field $\mathbb{F}_{\ell}(f)$, which is generated by the coefficients of $f$ over $\mathbb{F}_{\ell}$.

Let $a_{i}(f)$ be the $i$-th coefficient of the Fourier expansion of $f$. To show (2.6) it is enough to show that $a_{k}(f)-\tau(k)$ is always equal to zero, or there exists at least one nonzero element among the $a_{i}(f)-\tau(i)$ with $1 \leq i \leq \ell+2$.

If $f$ is congruent to $\Delta(q) \bmod \ell$, then $a_{k}(f)-\tau(k)$ is always equal to zero. Now suppose $f$ is not congruent to $\Delta(q)$ modulo $\ell$ and $a_{i}(f)-\tau(i)=0$ for $1 \leq i \leq \ell+2$. Then Proposition 4.10(b) of 14 together with Theorem 3.5(a) of 1 imply that $f$ comes from a level one newform of weight $k_{1}$ with $k_{1} \leq 2 \ell$. Since for any prime $p$, $\tau\left(p^{2}\right)=\tau(p)^{2}-p^{11}$ and $a_{p^{2}}(f)=a_{p}(f)^{2}-p^{k_{1}-1}$, we have $p^{k_{1}-12} \equiv 1 \bmod \ell$ for all primes $p$ satisfying $p^{2} \leq \ell+2$. We claim that $k_{1}-12 \equiv 0 \bmod (\ell-1)$. It suffices to show that the set of primes $\left\{p: p^{2} \leq \ell+2\right\}$ generates $\mathbb{F}_{\ell}^{*}$. If $\log _{2}(\ell-1) \geq 24$, then the main theorem of [15] promises that there exists a primitive root $\bmod \ell$ that is less than $\ell^{0.499}$. For $13 \leq \ell<2^{24}+1$, our claim is verified by exact calculation.

Now, let $A(q)=1$ be the Hasse invariant; then $f_{1}:=A(q)^{\left(k_{1}-12\right) /(\ell-1)} \Delta(q)$ is a newform of level one weight $k_{1}$, and $a_{k}\left(f_{1}\right)=a_{k}(f)$ for all $1 \leq k \leq \ell+2$. Since $\frac{k_{1}+1}{12}<\ell+2$, a theorem of Sturm [29] (or see [8]) says that $f_{1}$ is congruent to $f$, that is, $\Delta(q)$ is congruent to $f$ modulo $\ell$, which is a contradiction. So the proposition is proved.

As we have already seen, $\ell$ and a subset of $\left\{T_{k}-\tau(k): 1 \leq k \leq \ell+2\right\}$ may also suffice to generate $\mathfrak{m}$, so we introduce the optimal subset as follows. 
Definition 2.3. Let $\mathcal{S}$ be a set of positive integers such that $\ell$ and $T_{n}-\tau(n), n \in \mathcal{S}$, generate $\mathfrak{m}$. A set $\mathcal{S}$ is called optimal if $\prod_{n \in \mathcal{S}} n$ is minimal among all the $\mathcal{S}$.

In practice only those operators in the optimal subset need to be considered, which will accelerate the algorithm.

Let $n \geq 2$ be a prime not equal to $\ell$, and $Q$ a point of $X_{1}(\ell)_{\mathbb{F}_{p}}\left(\overline{\mathbb{F}}_{p}\right)$ represented by $\left(E_{(b, c)},(0,0)\right)$. The computation of $T_{n}(Q)$ comes down to computing isogenies of elliptic curves over some finite extension fields of $\mathbb{F}_{p}$,

$$
T_{n}\left(E_{(b, c)},(0,0)\right)=\sum_{C}\left(E_{(b, c)} / C,(0,0)+C\right),
$$

where $C$ runs over all the order $n$ subgroups of $E_{(b, c)}$. The following result about the complexity of computing $n$-isogeny is from 6 , where $n$ is small compared to the characteristic of the field.

Proposition 2.4. Let $\mathbb{F}_{q}$ be a finite field of characteristic $p, n$ a prime not equal to $p$, and $E$ and $\tilde{E}$ two elliptic curves over $\mathbb{F}_{q}$ in Weierstrass form. Assume there is a normalized isogeny $\phi: E \rightarrow \tilde{E}$ of degree $n$; then $\phi$ can be computed in $O\left(n^{1+\epsilon}\right)$ multiplications in the field $\mathbb{F}_{q}$.

We give two notices here: The first is that the original elliptic curve $E_{(b, c)}$ is defined over some finite field $\mathbb{F}$, but the isogeny may lie in an extension field of $\mathbb{F}$. The exact definition field of the isogeny can be obtained by computing the $n$-th classical modular polynomial $\Phi_{n}(X, Y)$ and solving the equation $\Phi_{n}\left(X, j\left(E_{(b, c)}\right)\right)=$ 0 , so the extension degree is less than or equal to the degree of $\Phi_{n}\left(X, j\left(E_{(b, c)}\right)\right)$. The second is that, in general, the isogenous curve $\tilde{E}$ is not in Tate normal form (2.1). Using the map $\phi$, or rather the point $\phi((0,0)) \in \tilde{E}, \tilde{E}$ can be transformed into Tate normal form after some coordinate changes. This gives a new point on the modular curve $X_{1}(\ell)_{\mathbb{F}_{p}}$.

\section{Computing in the Jacobian OF MOdular CURVES}

Let $\mathbb{F}_{q}$ be a finite extension $\mathbb{F}_{p}$ and $X_{1}(\ell)_{\mathbb{F}_{q}}$ the base change of $X_{1}(\ell)_{\mathbb{F}_{p}}$ to $\mathbb{F}_{q}$. One of the most important tasks of our algorithm is computing in the Jacobian $J_{1}(\ell)_{\mathbb{F}_{q}}$ of $X_{1}(\ell)_{\mathbb{F}_{q}}$. For general curves, we already have polynomial time algorithms to perform an operation (addition and subtraction) in their Jacobians [18, [31, [16], 22. For the Jacobian of modular curve, Couveignes uses the Brill-Noether algorithm to do the computation [9], while Bruin uses Khuri-Makdisi's algorithm [7]. We choose Heß's algorithm, with the advantage that it's easy to know the correspondences between points of the modular curve and places of its function field.

Let's recall the main idea of Heß's algorithm; for the details see [16]. Let $\mathbb{K}=$ $\mathbb{F}_{q}(x)[y] /(f(x, y))$ be the function field of $X_{1}(\ell)_{\mathbb{F}_{q}}$. There are isomorphisms

$$
J_{1}(\ell)\left(\mathbb{F}_{q}\right) \cong \operatorname{Pic}^{0}\left(X_{1}(\ell)_{\mathbb{F}_{q}}\right) \cong \mathrm{Cl}^{0}(\mathbb{K}) .
$$

Notice that there are some calculations behind the second isomorphism, i.e. computing the change of representations. Heß's algorithm is based on the arithmetic of the function field $\mathbb{K}$. Let $\mathbb{P}$ be the set of all places of $\mathbb{K}$ and $S$ the set of the places of $\mathbb{K}$ over the infinite place $\infty$ of $\mathbb{F}_{q}(x)$. The ring of elements of $\mathbb{K}$ being integral at all places of $S$ and $\mathbb{P} \backslash S$ are denoted by $\mathcal{O}_{S}$ and $\mathcal{O}^{S}$, respectively. They are Dedekind domains, called infinite and finite orders of $\mathbb{K}$, whose divisor groups are denoted by $\operatorname{Div}\left(\mathcal{O}_{S}\right)$ and $\operatorname{Div}\left(\mathcal{O}^{S}\right)$, respectively. The place of $\mathbb{K}$ corresponds to 
the prime ideal of $\mathcal{O}_{S}$ or $\mathcal{O}^{S}$. In fact, let $\operatorname{Div}(\mathbb{K})$ be the divisor group of $\mathbb{K}$; then $\operatorname{Div}(\mathbb{K})$ can be decomposed as 16

$$
\operatorname{Div}(\mathbb{K}) \stackrel{\sim}{\rightarrow} \operatorname{Div}\left(\mathcal{O}_{S}\right) \times \operatorname{Div}\left(\mathcal{O}^{S}\right) .
$$

Notice that the plane model of $X_{1}(\ell)$ given in [30] has singularities above $x=0$ and $x=-1$. We can check that places of $\mathbb{K}$ over the places $(x),(x+1)$ and $\left(\frac{1}{x}\right)$ of $\mathbb{F}_{q}(x)$ are cusps of $X_{1}(\ell)$. We will discuss how to compute the action of Hecke operators on these places in Section 3.2.

Now, let's focus on how to compute the action of Hecke operators on a place corresponding to a set of smooth points of $f(x, y)=0$. Such a place $\wp$ can be represented by two elements of $\mathbb{K}^{\times}$, which can be normalized as $f_{1}(x)=x^{d}+$ $a_{d-1} x^{d-1}+\ldots+a_{1} x+1$ and $f_{2}(x, y)=y^{m}+b_{m-1}(x) y^{m-1}+\ldots+b_{1}(x) y+b_{0}(x)$, where $a_{i}, 0 \leq i \leq d-1$, belong to the constant field $\mathbb{F}_{q}$, and $b_{i}(x), 0 \leq i \leq m-1$, are elements of $\mathbb{F}_{q}[x]$, with degrees less than $d$. So the point set corresponding to $\wp$ can be computed as follows. Let $f_{1}(x)$ and $f_{2}(x, y)$ be normalized generators of $\wp$. We first compute the roots of $f_{1}(x)=0$, denoted by $x_{i}, 1 \leq i \leq d$. Then, for each root $x_{i}$, compute the roots of $f_{2}\left(x_{i}, y\right)=0$, denoted by $y_{i j}, 1 \leq j \leq m$. The point set corresponding to $\wp$ is $\left\{\left(x_{i}, y_{i j}\right): 1 \leq i \leq d, 1 \leq j \leq m\right\}$. Recall that every point $\left(x_{i}, y_{i j}\right)$ satisfies $f\left(x_{i}, y_{i j}\right)=0$. Conversely, given a point set $\left\{\left(x_{i}, y_{i j}\right)\right.$ : $1 \leq i \leq d, 1 \leq j \leq m\}$, two generators for the corresponding prime ideal $\wp$ of $\mathcal{O}^{S}$ can be computed as follows. The first generator is clear, which is $f_{1}(x)=\prod_{i=1}^{d}\left(x-x_{i}\right)$. The second one can be recovered as follows: Let $b_{i}(x)=\sum_{k=0}^{d-1} c_{i k} x^{k}, 0 \leq i \leq m-1$, where $c_{i k}$ are parameters belong to $\mathbb{F}_{q}$; after interpolating the points $\left(x_{i}, y_{i j}\right)$, we have linear equations of $m d$ variables; the second generator can be known by solving these equations.

So, a degree $d$ place $\wp$ as in the above corresponds to a point set, denoted by $\left\{\left(x_{i}, y_{i}\right): 1 \leq i \leq d\right\}$, which forms a complete $\operatorname{Gal}\left(\mathbb{F}_{q^{d}} / \mathbb{F}_{q}\right)$-conjugate set. Using the coordinate transformation formulae (2.3), for each point $\left(x_{i}, y_{i}\right)$, the corresponding point on $X_{1}(\ell)_{\mathbb{F}_{p}}$ of the form $\left(E_{\left(b_{i}, c_{i}\right)},(0,0)\right)$ is clear, where $E_{\left(b_{i}, c_{i}\right)}$ is an elliptic curve in Tate normal form, and $(0,0)$ is a point of order $\ell$. As discussed in Section 2 , the action of Hecke operator $T_{n}$ on each point $\left(E_{\left(b_{i}, c_{i}\right)},(0,0)\right)$ leads to a sequence of elliptic curves. Using the inverse transformation formulae these curves give the point sets on the affine curve $f(x, y)=0$. Further, we have the corresponding places of the function field $\mathbb{K}$.

Let $P$ be a place of $\mathbb{K}=\mathbb{F}_{q}\left(X_{1}(\ell)\right)$, which is not equal to any of the cusps of $X_{1}(\ell)$, and $D$ a divisor of $\mathbb{K}$ consisting of such places. Then we define

Definition 3.1. Let $P, D$ be as above and $T_{n}$ a Hecke operator. $T_{n}(P)$ is defined to be the divisor of $\mathbb{K}$, corresponding to the point set $\sum_{i=1}^{d} T_{n}\left(E_{\left(b_{i}, c_{i}\right)},(0,0)\right)$, which is effective of degree $\Psi(n) d$, where $\Psi(n)=n \prod_{p \mid n}\left(1+\frac{1}{p}\right)$. Decompose $D$ as $\sum_{i=1}^{m} a_{i} P_{i}$, where $P_{i}$ are places; then $T_{n}(D)$ is defined to be $\sum_{i=1}^{m} a_{i} T_{n}\left(P_{i}\right)$.

Let $D_{0}$ be a fixed degree one place of $\mathbb{K}$, which will be served as an origin. Every element of $\mathrm{Cl}^{0}(\mathbb{K})$ can be represented by $D-g D_{0}$, where $g$ is the genus of $\mathbb{K}$ and $D$ is an effective divisor of degree $g$. Addition in $\mathrm{Cl}^{0}(\mathbb{K})$ means that, given effective divisors $A$ and $B$ of degree $g$, find an effective divisor $D$ of degree $g$, such that $D-g D_{0}$ is linearly equivalent to $A-g D_{0}+B-g D_{0}$. The complexity of such an operation can be found in [16]. 
Proposition 3.2. Notation is as above. There exists a constant $\omega \in[2,4]$ such that the divisor $D$ can be computed in $O\left(g^{\omega}\right)$ multiplications in the field $\mathbb{F}_{q}$, i.e. $O\left(g^{\omega} \log ^{1+\epsilon} q\right)$ bit operations.

Remark 3.3. We have not yet seen the precise value of $\omega$. However, using KhuriMakdisi's algorithm the complexity of a single group operation is known, i.e. $\omega=$ 2.376 , when fast algorithms for the linear algebra are used.

The following definition in 16 is very useful in our algorithm.

Definition 3.4. Let $A$ be a divisor with $\operatorname{deg}(A) \geq 1$. A divisor $\tilde{D}$ is called maximally reduced along $A$ if $\tilde{D} \geq 0$ and $\operatorname{dim}(\tilde{D}-r A)=0$ holds for all $r \geq 1$. Now let $D$ be any divisor and $\tilde{D}$ a divisor maximally reduced along $A$ such that $D$ is linearly equivalent to $\tilde{D}+r A$ for some $r \in \mathbb{Z}$. Then $\tilde{D}$ is called a reduction of $D$ along $A$.

If $\operatorname{deg}(A)=1$, then the reduction divisor $\tilde{D}$ is effective and unique.

3.1. Searching for an $\ell$-torsion point. One of the main steps in computing the Ramanujan subspace $V_{\ell} \bmod p$ is to find an $\ell$-torsion point in $J_{1}(\ell)$. In order to do that we have to work with a large enough extension field $\mathbb{F}_{q} / \mathbb{F}_{p}$, such that $J_{1}(\ell)\left(\mathbb{F}_{q}\right)$ contains $\ell$-torsion points. A direct way to get such a point is to pick a random point $Q_{0}$ in $J_{1}(\ell)\left(\mathbb{F}_{q}\right)$ and then compute $Q_{1}:=N_{\ell} Q_{0}$, where $N_{\ell}$ is the prime-to- $\ell$ part of $\# J_{1}(\ell)\left(\mathbb{F}_{q}\right)$. If $Q_{1}$ is nonzero and $\ell$-torsion, then we succeed. Otherwise, try $Q_{2}:=\ell Q_{1}$, check again, and after several steps, we can obtain a nonzero $\ell$-torsion point. As $\# J_{1}(\ell)\left(\mathbb{F}_{q}\right)$ is bounded above by $\mathrm{O}\left(q^{g}\right)$, using fast exponentiation, the running time of getting an $\ell$-torsion point is about $\log \left(q^{g}\right) \cdot \mathrm{O}\left(g^{\omega}\right)=\mathrm{O}\left(g^{1+\omega} \log q\right)$ multiplications in the field $\mathbb{F}_{q}$ or $\mathrm{O}\left(g^{1+\omega} \log ^{2+\epsilon} q\right)$ bit operations. The computation is costly, due to the huge factor $N_{\ell}$, which is nearly $q^{g}$. However, we can accelerate the calculation by introducing some tricks.

For each newform $f$ in $S_{2}\left(\Gamma_{1}(\ell)\right)$, there is an associated abelian variety $A_{f}$, and $\prod_{f} A_{f}$ is an isogeny decomposition of $J_{1}(\ell)$, where $f$ runs through a set of representatives for the Galois conjugacy classes of newforms in $S_{2}\left(\Gamma_{1}(\ell)\right)$. Let $f_{\ell}$ be the newform, which is congruent to $\Delta(q)$ modulo $\ell$. Then the Ramanujan subspace $V_{\ell}$ lands inside $A_{f_{\ell}}$. Let $A^{\prime}:=\prod_{f}^{\prime} A_{f}$ be the product of the abelian varieties, except $A_{f_{\ell}}$. The minimal polynomial of the Hecke operator $T_{2}$ acting on $A^{\prime}$ is denoted by $P_{2}[X] \in \mathbb{Z}[X]$. Now for a random point $Q_{0}$ in $J_{1}(\ell)\left(\mathbb{F}_{q}\right)$, $Q_{1}:=P_{2}\left(T_{2}\right)\left(Q_{0}\right)$ is a point in $A_{f_{\ell}}\left(\mathbb{F}_{q}\right)$. Similarly, let $n_{\ell}$ be the prime-to- $\ell$ part of $\# A_{f_{\ell}}\left(\mathbb{F}_{q}\right)$. By computing $n_{\ell} Q_{1}$, we can obtain an $\ell$-torsion point of $J_{1}(\ell)\left(\mathbb{F}_{q}\right)$. Since the calculation of $P_{2}\left(T_{2}\right)\left(Q_{0}\right)$ is easy and $n_{\ell}$ is smaller than $N_{\ell}$, which is bounded above by $q$ to the dimension of $A_{f_{\ell}}$, we can accelerate the algorithm, especially when the dimension of $A_{f_{\ell}}$ is small compared to $g$. However, it seems hard to get a theoretical bound of the dimension of $A_{f_{\ell}}$. We remark here several small examples:

\begin{tabular}{ccccccccccccc}
\hline Level $\ell$ & 13 & 17 & 19 & 29 & 31 & 37 & 41 & 43 & 47 & 53 & 59 & 61 \\
\hline $\operatorname{dim} J_{1}(\ell)$ & 2 & 5 & 7 & 22 & 26 & 40 & 51 & 57 & 70 & 92 & 117 & 126 \\
\hline $\operatorname{dim} A_{f_{\ell}}$ & 2 & 4 & 6 & 12 & 4 & 18 & 6 & 36 & 66 & 48 & 112 & 8 \\
\hline $\operatorname{dim} J_{H}(\ell)$ & & & & & 6 & & 11 & & & & & 26 \\
\hline
\end{tabular}


Another method, suggested by Maarten Derickx, works perfectly when the level $\ell$ satisfies $\ell \equiv 1 \bmod 10$. More precisely, let $\chi$ be the Dirichlet character associated to the newform $f_{\ell}$. Then we have for any prime $p \neq \ell, \chi(p) \cdot p \equiv p^{11} \bmod \ell$, and hence $\chi\left(p^{\frac{\ell-1}{10}}\right) \equiv p^{\ell-1} \equiv 1 \bmod \ell$. This means that $f_{\ell}$ is invariant under the action of diamond operators in the cyclic subgroup $H=\left\{p^{\frac{\ell-1}{10}}: p \neq \ell\right.$ prime $\}$ of $G=(\mathbb{Z} / \ell \mathbb{Z})^{\times}$. The Ramanujan subspace $V_{\ell}$ lands inside the Jacobian variety $J_{H}(\ell)$, where $J_{H}(\ell)$ is isogenous to the Jacobian of the modular curve $X_{H}(\ell)$ associated to the subgroup of $\mathrm{SL}_{2}(\mathbb{Z})$ of matrices $[a, b ; c, d]$ with $c$ divisible by $\ell$ and $a$ in $H$ modulo $\ell$. We can apply the algorithm to the modular curve $X_{H}(\ell)$ and the Jacobian variety $J_{H}(\ell)$ instead of $X_{1}(\ell)$ and $J_{1}(\ell)$, respectively. This useful observation enables us to carry out the calculation of the level $\ell=31$ case.

We now explain how to compute $\# J_{1}(\ell)\left(\mathbb{F}_{q}\right)$.

Lemma 3.5 (Manin, Shokurov, Merel, Cremona). For $\ell$ a prime and $p \notin\{5, \ell\}$ another prime, the Zeta function of $X_{1}(\ell)_{\mathbb{F}_{p}}$ can be computed in deterministic polynomial time in $\ell$ and $p$.

Remark 3.6. Given this Zeta function we can easily compute $\# J_{1}(\ell)\left(\mathbb{F}_{q}\right)$, for $q$ is a power of $p$; see [9]. Similarly, we can compute $\# A_{f}\left(\mathbb{F}_{q}\right)$ in deterministic polynomial time in $\ell$ and $p$, by applying the algorithm to newforms in $[f]$, where $[f]=\left\{f^{\sigma}\right.$ : $\sigma \in \operatorname{Gal}(\overline{\mathbb{Q}} / \mathbb{Q})\}$.

3.2. Distinguishing the rational cusps. In our algorithm, we will compute the action of Hecke operator $T_{n}$ on points of $J_{1}(\ell)$. Especially, we need to know the action of $T_{n}$ on the $\mathbb{Q}$-rational cusps of $X_{1}(\ell)$. Notice that $T_{n}, n \in \mathbb{Z}^{+}$, is defined over $\mathbb{Q}$, so it maps $\mathbb{Q}$-rational point to $\mathbb{Q}$-rational point. As far as we know, there is no easy way to know the action directly from only the places $O_{i}, i \in\left\{1, \ldots, \frac{\ell-1}{2}\right\}$. But, a coset representative of the $\mathbb{Q}$-rational cusps of $X_{1}(\ell)$ is clear, i.e. $\left\{\frac{1}{1}, \frac{1}{2}, \ldots, \frac{1}{(\ell-1) / 2}\right\}$. For prime $n \neq \ell$, we have $T_{n}\left(\frac{1}{m}\right)=\frac{1}{m}+n \frac{1}{n m}$, where $\overline{n m}$ is the class of integer $n m$ in $(\mathbb{Z} / \ell \mathbb{Z})^{*} /\{ \pm 1\}$. So knowing the 1-1 correspondence between $\left\{\frac{1}{i}, 1 \leq i \leq \frac{\ell-1}{2}\right\}$ and $\left\{O_{i}, 1 \leq i \leq \frac{\ell-1}{2}\right\}$ leads to knowing the action of $T_{n}$ on $O_{i}$. In general, it's not easy to know the correspondence [23. Our strategy is to reduce the problem to a finite field, as follows. Choose a prime $p$, such that $\# J_{1}(\ell)\left(\mathbb{F}_{p}\right)$ has a small factor $d$, and let $g$ be the genus of the function field $\mathbb{F}_{p}\left(X_{1}(\ell)\right)$. Fix a cusp $O_{i}$ served as the origin. As discussed above, let $D-g O_{i}$ be a degree zero random divisor of order $d$. In general $D$ doesn't contain cusps; if it does, try a new one. Now, assume $O_{i}$ corresponds to $\frac{1}{m}$ for some $m \in\left\{1, \ldots, \frac{\ell-1}{2}\right\}$ and $\frac{1}{\overline{n m}}$ corresponds to $O_{j}$ for some $j \in\left\{1, \ldots, \frac{\ell-1}{2}\right\}$. Then, for prime $n$, compute $D_{n}:=T_{n}\left(D-g O_{i}\right)$ by the assumption as follows:

$$
D_{n}=T_{n}(D)-g T_{n}\left(O_{i}\right)=T_{n}(D)-g\left(O_{i}+n O_{j}\right) .
$$

If $D_{n}$ is not of order $d$, then the assumption is wrong. Replace $O_{i}$ by another $\frac{1}{m}$, or $\frac{1}{\overline{n m}}$ by another $O_{j}$, and try again. The complete correspondence can be detected after several tries 1 In fact, the correspondence is known up to cyclic permutation, but it is enough for our algorithm. There is one more thing that should be noticed: the degree 0 divisors of the form $O_{i}-O_{j}, 1 \leq i, j \leq \frac{\ell-1}{2}$, generate a subgroup of $J_{1}(\ell)\left(\mathbb{F}_{p}\right)$, which is called cuspidal subgroup, so the chosen factor $d$ should not be a multiple of the order of the cuspidal subgroup.

\footnotetext{
${ }^{1}$ We can use the diamond operators instead, which is faster, as suggested by Maarten Derickx.
} 
So now we know the action of the Hecke operator on $\mathrm{Cl}^{0}(\mathbb{K})$ explicitly. The complexity of computing $T_{n}(Q)$ can be determined as follows, where $Q$ is a degree zero $\mathbb{F}_{q}$-divisor of the form $\sum_{i=1}^{m} a_{i} P_{i}-g O, O$ is fixed to be the cusp $O_{1}$ and $n$ is a prime not equal to $\ell$.

As mentioned above, we first compute the point set of the degree $d_{i}$ place $P_{i}$ and pick one of them that forms a point $\left(E_{\left(b_{i}, c_{i}\right)},(0,0)\right)$ on the modular curve, which is defined over $\mathbb{F}_{q^{d_{i}}}$. Denote the factorization of $\Phi_{n}\left(X, j\left(E_{\left(b_{i}, c_{i}\right)}\right)\right)$ over $\mathbb{F}_{q^{d_{i}}}[X]$ as $\prod_{j=1}^{h} F_{j}(X)$, with $F_{j}(X)$ irreducible of degree $f_{j}$. Now for each root of $F_{j}(X)=0$, there is an isogeny of degree $n$ defined over $\mathbb{F}_{q^{d_{i}} f_{j}}$. Computing this isogeny takes

$$
\mathrm{O}\left(n^{1+\epsilon} \cdot\left(\log q^{d_{i} f_{j}}\right)^{1+\epsilon}\right)=\mathrm{O}\left(\left(n d_{i} f_{j} \log q\right)^{1+\epsilon}\right)
$$

bit operations.

Notice that isogenous curves corresponding to the roots of $F_{j}(X)$ form a $\operatorname{Gal}\left(\mathbb{F}_{q^{d_{i} f_{j}}} / \mathbb{F}_{q^{d_{i}}}\right)$-conjugate set. So it suffices to compute any one of them. So the complexity of computing $T_{n}\left(\left(E_{\left(b_{i}, c_{i}\right)},(0,0)\right)\right)$ is about

$$
C_{i}:=\sum_{j=1}^{h} \mathrm{O}\left(\left(n d_{i} f_{j} \log q\right)^{1+\epsilon}\right) .
$$

Since $\sum_{j=1}^{h} f_{j}=n+1, C_{i}$ is bounded above by $\mathrm{O}\left(\left(n d_{i} n \log q\right)^{1+\epsilon}\right)$.

The complexity of computing $T_{n}(Q)$ is $C:=\sum_{i=1}^{m} C_{i}$. Since $\sum_{i=1}^{m} a_{i} d_{i}=g, C$ is bounded above by $\mathrm{O}\left(\left(n^{2} g \log q\right)^{1+\epsilon}\right)$ bit operations.

Notice that after the action of $T_{n}$, the divisor $T_{n}(Q)$ becomes complicated. We would like to simplify it before going into further calculation. In general, the reduction of $T_{n}(Q)$ along the degree one divisor $O$ comes down to performing at most $n$ additions in the Jacobian, with a complexity of $\mathrm{O}\left(n g^{\omega} \log ^{1+\epsilon} q\right)$ bit operations.

\section{Computing the coefficients of modular forms}

After some modification, the simplified algorithm proposed in [9] can be used to compute the Ramanujan subspace $V_{\ell} \bmod p$ efficiently. We can even give a complexity analysis of the algorithm. Roughly speaking, our algorithm for computing the Ramanujan tau function works as follows.

Algorithm 4.1. Computing the Ramanujan tau function modulo a prime $\ell$.

Input: A plane model for $X_{1}(\ell)$ and a prime $q$.

Output: $\tau(q) \bmod \ell$.

(1) Computing the Ramanujan subspace $V_{\ell}$ modulo a prime $p$.

(1.1) Determine the minimal extension $\mathbb{F}_{p^{e}} / \mathbb{F}_{p}$ such that $V_{\ell} \bmod p \subset$ $J_{1}(\ell)\left(\mathbb{F}_{p^{e}}\right)$.

(1.2) Pick a random point $Q$ in $J_{1}(\ell)\left(\mathbb{F}_{p^{e}}\right)$.

(1.3) Construct an $\ell$-torsion point $R$ by multiplying $Q$ by a suitable factor of $\# J_{1}(\ell)\left(\mathbb{F}_{p^{e}}\right)$.

(1.4) Get a point of $V_{\ell} \bmod p$ by projecting $R$ into $V_{\ell} \bmod p$ using Hecke operators.

(1.5) Collect a set of points in $V_{\ell} \bmod p$ using steps (1.2)-(1.4) and find a basis of $V_{\ell} \bmod p$.

(2) Reconstruct the Ramanujan subspace $V_{\ell} / \mathbb{Q}$ from sufficiently many $V_{\ell} \bmod p$ by the Chinese Remainder Theorem. 
(3) Compute the mod-l representation $\rho_{\ell}: G a l(\overline{\mathbb{Q}} / \mathbb{Q}) \rightarrow G L_{2}\left(\mathbb{F}_{\ell}\right)$ associated to $\Delta(q) \bmod \ell$ by using Hensel lifting method and Dokchitser's algorithm.

(4) Compute $\tau(q) \bmod \ell$ by the formula $\tau(q) \equiv \operatorname{Tr}\left(\rho_{\ell}\left(\right.\right.$ Frob $\left.\left._{q}\right)\right) \bmod \ell$.

The following sections are devoted to the details of Algorithm 4.1

4.1. Computing the Ramanujan subspace modulo $p$. The characteristic polynomial of the Frobenius endomorphism Frob $p$ acting on the Ramanujan subspace $V_{\ell} \bmod p$ is $X^{2}-\tau(p) X+p^{11} \bmod \ell$ and the field of definition of each point of $V_{\ell} \bmod p$ is an extension of $\mathbb{F}_{p}$ with degree denoted by $d_{p}$. Thus we have

$$
d_{p} \leq \min _{t}\left\{t: X^{t} \equiv 1 \bmod \left(X^{2}-\tau(p) X+p^{11}, \ell\right), t \geq 1\right\} .
$$

For convenience, we define,

Definition 4.2. With notations as above, a prime $p$ is called $s$-good if $d_{p}<\ell$.

Primes with small $d_{p}$ are preferable to accelerate the calculation. In fact, all of our current calculation uses primes with $d_{p} \leq 4$. We now use Chebotarev's density theorem to study the density of $s$-good primes. Let $K / \mathbb{Q}$ be a finite Galois extension and $C$ a union of conjugacy classes of $G:=\operatorname{Gal}(K / \mathbb{Q})$. For $x>2$ a real number, let $\pi_{C}(x, K)$ denote

$$
\pi_{C}(x, K):=\#\left\{p: p<x \text { prime unramified in } K \text { and }\left[\frac{K / \mathbb{Q}}{p}\right] \subset C\right\},
$$

where $\left[\frac{K / \mathbb{Q}}{p}\right]$ denotes the Artin symbol representing the conjugacy class of the Frobenius above $p$ in $G$. The Chebotarev density theorem asserts that

$$
\pi_{C}(x, K)=\frac{|C|}{|G|} \pi(x), x \rightarrow+\infty,
$$

where $\pi(x)$ denotes the number of primes less than $x$. The first effective version of Chebotarev's density theorem, proved by Lagarias and Odlyzko 24 and refined by Serre ([27, page 133), states that, assuming the generalised Riemann hypothesis for number fields $(\mathrm{GRH})$, there exists an absolute constant $c_{6}$ such that

$$
\left|\pi_{C}(x, K)-\frac{|C|}{|G|} \operatorname{Li}(x)\right| \leq c_{6} \frac{|C|}{|G|} x^{\frac{1}{2}}\left(\log d_{K}+n_{K} \log x\right),
$$

where $\operatorname{Li}(x):=\int_{2}^{x}(1 / \log y) d y, d_{K}$ is the discriminant of $K$ and $n_{K}:=[K: \mathbb{Q}]$. Now let $\pi_{s}(x)$ be the number of $s$-good primes less than $x$, and we have the following proposition.

Proposition 4.3. Let $\ell \geq 11$ be a nonexceptional prime for $\Delta(q)$ and $\rho_{\ell}: \operatorname{Gal}(\overline{\mathbb{Q}} / \mathbb{Q})$ $\rightarrow \mathrm{GL}_{2}\left(\mathbb{F}_{\ell}\right)$ the mod- $\ell$ representation associated to $\Delta(q)$. Denote $K_{\ell}$ the fixed field of $\operatorname{ker} \rho_{\ell}$. We have

$$
\lambda:=\lim _{x \rightarrow+\infty} \frac{\pi_{s}(x)}{\pi(x)} \geq \frac{\ell^{3}-\ell^{2}-2 \ell+2}{2\left(\ell^{3}-\ell\right)} .
$$

Assuming GRH, we have

$$
\left.\left|\pi_{s}(x)-\lambda \cdot \operatorname{Li}(x)\right| \leq c_{6} \cdot \lambda \cdot x^{\frac{1}{2}}\left(\log d_{K_{\ell}}+n_{K_{\ell}} \cdot \log x\right)\right),
$$

where $d_{K_{\ell}}$ is the discriminant of $K_{\ell}, n_{K_{\ell}}=\left[K_{\ell}: \mathbb{Q}\right]$ and $c_{6}$ is an absolute constant. 
Proof. The representation $\rho_{\ell}$ factors through $\rho_{\ell}: \operatorname{Gal}\left(K_{\ell} / \mathbb{Q}\right) \rightarrow \mathrm{GL}_{2}\left(\mathbb{F}_{\ell}\right)$, which is unramified outside $\ell$. Now, for a prime $p$ not equal to $\ell$, we have $\rho_{\ell}\left(\operatorname{Frob}_{p}\right) \in$ $\mathrm{GL}_{2}\left(\mathbb{F}_{\ell}\right)$ and $d_{p}$ is the order of the matrix $\rho_{\ell}\left(\right.$ Frob $\left._{p}\right)$. By the Chebotarev density theorem, for any conjugacy class $C$ of $G:=\operatorname{Gal}\left(K_{\ell} / \mathbb{Q}\right)$ we have

$$
\pi_{C}\left(x, K_{\ell}\right)=\frac{|C|}{|G|} \pi(x), x \rightarrow+\infty .
$$

Define $\mathcal{C}=\bigcup_{\operatorname{ord}(C)<\ell} C$, where ord $(C)$ represents the order of any element in $C$. Then we have

$$
\lim _{x \rightarrow+\infty} \frac{\pi_{s}(x)}{\pi(x)}=\frac{|\mathcal{C}|}{|G|} .
$$

Hence we would like to know the image of the representation $\rho_{\ell}$.

Since $\ell$ is not exceptional, we have $\operatorname{Im}\left(\rho_{\ell}\right)=\left\{g \in \mathrm{GL}_{2}\left(\mathbb{F}_{\ell}\right): \operatorname{det}(g) \in\left(\mathbb{F}_{\ell}^{\times}\right)^{11}\right\}$. The representatives of conjugacy classes in $\mathrm{GL}_{2}\left(\mathbb{F}_{\ell}\right)$ are as follows:

$$
\begin{aligned}
& c_{1}(x):=\left[\begin{array}{cc}
x & 0 \\
0 & x
\end{array}\right], x \in \mathbb{F}_{\ell}^{\times}, \\
& c_{2}(x):=\left[\begin{array}{ll}
x & 1 \\
0 & x
\end{array}\right], x \in \mathbb{F}_{\ell}^{\times} \text {, } \\
& c_{3}(x, y):=\left[\begin{array}{ll}
x & 0 \\
0 & y
\end{array}\right], x \neq y \in \mathbb{F}_{\ell}^{\times}, c_{3}(x, y)=c_{3}(y, x) \text {, } \\
& c_{4}(z):=\left[\begin{array}{cc}
x & D y \\
y & x
\end{array}\right], z=x+\sqrt{D} y \in \mathbb{F}_{\ell^{2}} \backslash \mathbb{F}_{\ell}, c_{4}(z)=c_{4}(\bar{z}) \text {, where } \bar{z}:=x-\sqrt{D} y \text {. }
\end{aligned}
$$

Here $c_{3}(x, y)=c_{3}(y, x)$ means that the conjugacy classes of these two elements agree. Let $C_{1}(x)$ be the conjugacy class with representative $c_{1}(x)$. Then we have $\left|C_{1}(x)\right|=1, \operatorname{ord}\left(C_{1}(x)\right) \mid(\ell-1)$ and there are $N_{1}=\ell-1$ such classes. Similarly, we have $\left|C_{2}(x)\right|=\ell^{2}-1$, ord $\left(C_{2}(x)\right)\left|\ell(\ell-1), N_{2}=\ell-1 ;\right| C_{3}(x, y) \mid=\ell(\ell+1)$, $\operatorname{ord}\left(C_{3}(x, y)\right)\left|(\ell-1), N_{3}=\frac{1}{2}(\ell-1)(\ell-2) ;\right| C_{4}(z)\left|=\ell(\ell-1), \operatorname{ord}\left(C_{4}(z)\right)\right|\left(\ell^{2}-1\right)$, $N_{4}=\frac{1}{2} \ell(\ell-1)$. We can see $\left|C_{1}(x)\right| N_{1}+\left|C_{2}(x)\right| N_{2}+\left|C_{3}(x, y)\right| N_{4}+\left|C_{4}(z)\right| N_{4}=$ $\left|\mathrm{GL}_{2}\left(\mathbb{F}_{\ell}\right)\right|=(\ell-1)^{2} \ell(\ell+1)$.

The subgroup $\operatorname{Im}\left(\rho_{\ell}\right)$ consists of those conjugacy classes whose representative matrices have determinant in $\left(\mathbb{F}_{\ell}^{\times}\right)^{11}$. For example $\operatorname{Im}\left(\rho_{\ell}\right)$ contains conjuagcy classes with representatives $c_{1}(x)$ satisfying $x^{2} \in\left(\mathbb{F}_{\ell}^{\times}\right)^{11}$. Denote the number of such classes by $M_{1}$ and set $L:=\left|\left(\mathbb{F}_{\ell}^{\times}\right)^{11}\right|$. Then we have $M_{1}=L$. Similarly $M_{2}=L, M_{3}=\frac{1}{2} L(\ell-2)$ and $M_{4}=\frac{1}{2} L \ell$. So we have

$$
\frac{|\mathcal{C}|}{|G|} \geq \frac{M_{1}+M_{3} \cdot \ell(\ell+1)}{M_{1}+M_{2} \cdot\left(\ell^{2}-1\right)+M_{3} \cdot \ell(\ell+1)+M_{4} \cdot \ell(\ell-1)}=\frac{\ell^{3}-\ell^{2}-2 \ell+2}{2\left(\ell^{3}-\ell\right)} .
$$

The second statement follows from the effective version of Chebotarev's density theorem.

Now let $p$ be prime and $\mathbb{F}_{q}:=\mathbb{F}_{p^{d_{p}}}$; then $V_{\ell} \bmod p$ is a subgroup of $J_{1}(\ell)\left(\mathbb{F}_{q}\right)[\ell]$.

For every integer $n \geq 2$, the characteristic polynomial of $T_{n}$ acting on $S_{2}\left(\Gamma_{1}(\ell)\right)$ is a degree $g$ monic polynomial belonging to $\mathbb{Z}[X]$. We denote it by $A_{n}(X)$, which can be factored as

$$
A_{n}(X) \equiv B_{n}(X)(X-\tau(n))^{e_{n}} \bmod \ell,
$$

with $B_{n}(X)$ monic and $B_{n}(\tau(n)) \neq 0 \in \mathbb{F}_{\ell}$. The exponent $e_{n}$ is $\geq 1$ due to the theorem of congruence of modular forms (Theorem 2.5.7 of [13]). We call $\pi_{n}$ : $J_{1}(\ell)\left(\mathbb{F}_{q}\right)[\ell] \rightarrow J_{1}(\ell)\left(\mathbb{F}_{q}\right)[\ell]$ the projection map, which maps an $\ell$-torsion point $Q \in$ $J_{1}(\ell)\left(\mathbb{F}_{q}\right)[\ell]$ to an $\ell$-torsion point $B_{n}\left(T_{n}\right)(Q)$ of $J_{1}(\ell)\left(\mathbb{F}_{q}\right)[\ell]$, and maps bijectively $V_{\ell} \bmod p$ onto itself. Assume $E:=\pi_{n}(Q) \neq 0$ and define the exponent $f_{n}$ as the nonnegative integer satisfying

$$
\left(T_{n}-\tau(n)\right)^{f_{n}}(E) \neq 0 \text { and }\left(T_{n}-\tau(n)\right)^{f_{n}+1}(E)=0 .
$$


Then $f_{n}$ is in $\left[0, e_{n}\right)$, since $\left(T_{n}-\tau(n)\right)^{e_{n}}(E)=0$. Let $\tilde{\pi}_{n}$ be the composition map of $\pi_{n}$ and $\left(T_{n}-\tau(n)\right)^{f_{n}}$ and $\pi_{\mathcal{S}}:=\prod_{n \in \mathcal{S}} \tilde{\pi}_{n}$, where $\mathcal{S}$ is an optimal set defined in Definition 2.3. Then we have $\pi_{\mathcal{S}}(Q) \in V_{\ell} \bmod p$.

So, the complexity of finding a nonzero point in $V_{\ell} \bmod p$ can be determined as follows.

As described in Section 3, it takes $\mathrm{O}\left(g^{1+\omega} \log ^{2+\epsilon} q\right)$ bit operations to get an $\ell$ torsion point of $J_{1}(\ell)\left(\mathbb{F}_{q}\right)$. Denote the $\ell$-torsion point as $Q_{0}=D-g O$, where $D$ is an effective divisor of degree $g$. For $n \in \mathcal{S}$, the map $\tilde{\pi}_{n}$ can be written as $T_{n}^{d}+a_{d-1} T_{n}^{d-1}+\ldots+a_{1} T_{n}+a_{0}$, where $a_{i} \in \mathbb{F}_{\ell}$ and $d<g$. The divisor $\tilde{\pi}_{n}\left(Q_{0}\right)$ can be computed recursively, i.e. compute and simplify (as mentioned in Section 3.2) $Q_{i+1}:=T_{n}\left(Q_{i}\right)$ for $i=0, \ldots, d-1$. The complexity of each step is $\mathrm{O}\left(\left(n^{2} g \log q\right)^{1+\epsilon}\right)+\mathrm{O}\left(n g^{\omega} \log ^{1+\epsilon} q\right)$, which equals $\mathrm{O}\left(\ell g^{\omega} \log ^{1+\epsilon} q\right)$, since $n \in \mathrm{O}(\ell), g \in \mathrm{O}\left(\ell^{2}\right)$ and $\omega \in[2,4]$. As $d$ is in $\mathrm{O}(g)$, computing and simplifying $Q_{0}, \ldots, Q_{d}$ takes $d \cdot \mathrm{O}\left(\ell g^{\omega} \log ^{1+\epsilon} q\right)=\mathrm{O}\left(\ell g^{1+\omega} \log ^{1+\epsilon} q\right)$. Given $Q_{i}$, since $a_{i} \in \mathbb{F}_{\ell}$ the complexity of computing $Q_{d}+a_{d-1} Q_{d-1}+\ldots+a_{1} Q_{1}+a_{0} Q_{0}$ is bounded above by $d \ell \cdot \mathrm{O}\left(g^{\omega} \log ^{1+\epsilon} q\right)=\mathrm{O}\left(\ell g^{1+\omega} \log ^{1+\epsilon} q\right)$. In summary, the complexity of computing $\tilde{\pi}_{n}\left(Q_{0}\right)$ is still in $\mathrm{O}\left(\ell g^{1+\omega} \log ^{1+\epsilon} q\right)$. So the complexity of computing $\pi_{\mathcal{S}}\left(Q_{0}\right)=\prod_{n \in \mathcal{S}} \tilde{\pi}_{n}\left(Q_{0}\right)$ is bounded above by $\mathrm{O}\left(\ell^{2} g^{1+\omega} \log ^{1+\epsilon} q\right)$, since $|\mathcal{S}|<\ell$. So it takes

$$
\mathrm{O}\left(g^{1+\omega} \log ^{2+\epsilon} q\right)+\mathrm{O}\left(\ell^{2} g^{1+\omega} \log ^{1+\epsilon} q\right)=\mathrm{O}\left(g^{1+\omega} \log ^{1+\epsilon} q\left(\ell^{2}+\log q\right)\right)
$$

bit operations to get a point in $V_{\ell} \bmod p$.

For two nonzero random points $Q_{1}, Q_{2} \in J_{1}(\ell)\left(\mathbb{F}_{q}\right)[\ell]$, the probability that $\pi_{S}\left(Q_{1}\right)$ and $\pi_{S}\left(Q_{2}\right)$ are linearly independent is close to $1-\frac{1}{\ell}$. So after several attempts we will get a basis of $V_{\ell} \bmod p$. The complexity of checking linear independence of two elements in $V_{\ell} \bmod p$ is $\mathrm{O}\left(\ell g^{\omega} \log ^{1+\epsilon} q\right)$. Hence the complexity of getting a basis of $V_{\ell} \bmod p$ is the same as given in (4.1).

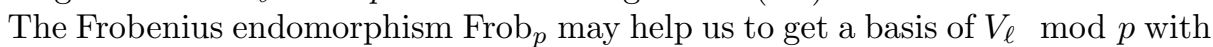
lower cost in some cases. Namely, if the characteristic polynomial $X^{2}-\tau(p) X+p^{11}$ $\bmod \ell$ is irreducible, then for any nonzero point $Q \in V_{\ell} \bmod p, \operatorname{Frob}_{p}(Q)$ and $Q$ are linearly independent. So $V_{\ell} \bmod p=\mathbb{F}_{\ell} Q+\mathbb{F}_{\ell}\left(\operatorname{Frob}_{p}(Q)\right)$. If $X^{2}-\tau(p) X+$ $p^{11} \bmod \ell$ has two different roots in $\mathbb{F}_{\ell}$, then for any nonzero point $Q \in V_{\ell} \bmod p$ the probability that $Q$ and $\operatorname{Frob}_{p}(Q)$ are linearly independent is $1-\frac{1}{\ell}$.

Remark 4.4. (1) In practice, the optimal set $\mathcal{S}$ contains only small primes, for example, $\mathcal{S}=\{2\}$ for $\ell \in\{13,17,19\}$. The algorithm takes the main effort to get an $\ell$-torsion point.

(2) If $p$ is an $s$-good prime, then $\log q=d_{p} \log p<\ell \log p$ and the complexity of computing $V_{\ell} \bmod p$ is

$$
\mathrm{O}\left(g^{1+\omega} \log ^{1+\epsilon} q\left(\ell^{2}+\log q\right)\right)=\mathrm{O}\left(\ell^{4+2 \omega+\epsilon} \log ^{1+\epsilon} p \cdot(\ell+\log p)\right) .
$$

If $p$ is not $s$-good, then $\log q=d_{p} \log p<\ell^{2} \log p$ and the complexity of computing $V_{\ell} \bmod p$ is

$$
\mathrm{O}\left(\ell^{6+2 \omega+\epsilon} \log ^{1+\epsilon} p \cdot(1+\log p)\right)=\mathrm{O}\left(\ell^{6+2 \omega+\epsilon} \log ^{2+\epsilon} p\right) .
$$

4.2. Computing the Ramanujan subspace. Fix a $\mathbb{Q}$-rational cusp $O$ of $X_{1}(\ell)$, which will be served as the origin of the Jacobi map. For every point $x \in V_{\ell}$, let $D$ be the reduction of $x$ along $O$, i.e. $D=x+d O$. Then $D$ is an effective divisor of degree $d$, which can be decomposed as $D=Q_{1}+\ldots+Q_{d}$; here $d$ is called the stability of $x$, denoted by $\theta(x)$. Choose a rational function $\psi(x) \in \mathbb{Q}\left(X_{1}(\ell)\right)$, which has no pole 
except at $O$, and define a function $\iota: V_{\ell} \rightarrow \overline{\mathbb{Q}}$ as $\iota(x)=\psi\left(Q_{1}\right)+\ldots+\psi\left(Q_{d}\right) \in \overline{\mathbb{Q}}$. From the uniqueness of $D$, we have $\iota(\sigma(x))=\sigma(\iota(x))$ for any $\sigma \in \operatorname{Gal}(\overline{\mathbb{Q}} / \mathbb{Q})$, which is very important. Let $\wp$ be a prime ideal of $\mathbb{Q}(x)$ over $p$. We hope that the uniqueness property still holds in the reduction world, which means the following: let $\tilde{x}, \tilde{O}, \tilde{D}$ and $\tilde{Q}_{i}$ be the reductions of $x, O, D$ and $Q_{i}$ modulo $\wp$ respectively. The reduction of $\tilde{x}$ along $\tilde{O}$ is denoted by $\tilde{E}=\tilde{x}+\theta(\tilde{x}) \tilde{O}$, where $\theta(\tilde{x}) \leq \theta(x)$. If $\theta(\tilde{x})=\theta(x)$, then by the uniqueness property, we have $\tilde{E}=\tilde{D}=\tilde{Q}_{1}+\ldots+\tilde{Q}_{d}$. As we don't know the value of $\theta(x)$ in advance, an algorithm to determine when $\theta(\tilde{x})=\theta(x), \forall x \in V_{\ell}$, is needed. In [7, Bruin gave such an algorithm and proved that, for at least half of the primes smaller than $\mathrm{O}\left(\ell^{\delta}\right)$, the following holds: $\theta(\tilde{x})=$ $\theta(x), \forall x \in V_{\ell}$; such primes are called $\mathfrak{m}$-good primes 2 In practice, since $\theta(x)$ is less than or equal to the genus of $X_{1}(\ell)$, if $\theta(\tilde{x})$ is equal to the genus for every $\tilde{x} \in V_{\ell}$ $\bmod p$, then $\theta(\tilde{x})=\theta(x)$ automatically for all $x \in V_{\ell}$. We remark here that our computation suggests that most of the primes are $\mathfrak{m}$-good.

Now for any $\mathfrak{m}$-good prime $p$, define

$$
P(X):=\prod_{x \in V_{\ell} \backslash O}(X-\iota(x)) .
$$

It is a polynomial in $\mathbb{Q}[X]$ of degree $\ell^{2}-1$, whose reduction modulo $p$ is exactly the polynomial

$$
\tilde{P}(X):=\prod_{\tilde{x} \in V_{\ell}}(X-\tilde{\bmod }(\tilde{x})),
$$

where $\tilde{\iota}$ is the reduction map of $\iota$.

Given $V_{\ell} \bmod p=\mathbb{F}_{\ell} e_{1}+\mathbb{F}_{\ell} e_{2}$, the computation of $\tilde{P}(X)$ comes down to performing $\ell^{2}$ additions in the Jacobian, with a complexity $\ell^{2} \cdot \mathrm{O}\left(g^{\omega} \log ^{1+\epsilon} q\right)=$ $\mathrm{O}\left(g^{1+\omega} \log ^{1+\epsilon} q\right)$.

The heights of coefficients of $P(X)$ are expected to be in $\mathrm{O}\left(\ell^{\delta}\right)$, for some absolute constant $\delta$. Using this fact, there exists a constant $c$ such that

$$
\prod_{p \leq L, \text { prime }} p>c \cdot \exp (L) .
$$

To recover $P(X)$ from the $\tilde{P}(X)$ 's, it suffices to take the upper bound $L$ of good primes to be $\mathrm{O}\left(\ell^{\delta}\right)$. So the complexity of computing $P(X)$ will be

$$
\sum_{p \leq L, \text { prime }} \mathrm{O}\left(\ell^{6+2 \omega+\epsilon} \log ^{2+\epsilon} p\right)=\mathrm{O}\left(\ell^{6+2 \omega+\delta+\epsilon}\right) .
$$

In practice, it would be better to choose the function $\psi(x) \in \mathbb{Q}\left(X_{1}(\ell)\right)$, such that the degree is equal to the gonality of the curve $X_{1}(\ell)$. For $\ell \leq 40, \psi(x)$ have been computed by Derickx and Hoeij; see [17] and [10.

Remark 4.5. If those primes less than $L$ as well as $s$-good and $\mathfrak{m}$-good suffice to recover $P(X)$, then the complexity is $\mathrm{O}\left(\ell^{5+2 \omega+\delta+\epsilon}\right)$.

4.3. Finding the Frobenius endomorphism. The Galois representation associated to $\Delta(q)$ is denoted by $\rho_{\ell}: \operatorname{Gal}(\overline{\mathbb{Q}} / \mathbb{Q}) \rightarrow \mathrm{GL}_{2}\left(\mathbb{F}_{\ell}\right)$. Let $K_{\ell}:=\overline{\mathbb{Q}}^{\text {ker } \rho_{\ell}}$ be the field cut out by the representation; then $K_{\ell}$ is the splitting field of the polynomial $P(X)$. For any prime $p \neq \ell$, the trace of Frobenius $\operatorname{Tr}\left(\rho_{\ell}\left(\right.\right.$ Frob $\left.\left._{p}\right)\right)$ is equal to $\tau(p) \bmod \ell$. So we would like to identify the conjugacy class of the $\operatorname{Galois} \operatorname{group} \operatorname{Gal}\left(K_{\ell} / \mathbb{Q}\right)$,

\footnotetext{
${ }^{2}$ Here we require that the constant $\delta$ satisfies $\sum_{p}$ is not m-good prime $\log p<\ell^{\delta}$.
} 
where the Frobenius Frob $_{p}$ lands inside. The algorithm described in [12] can be used perfectly to do the computation.

Let $\left(a_{i}\right)_{1 \leq i \leq \ell^{2}-1}$ be the roots of $P(X)$ in $K_{\ell}$ and $h(X)$ some polynomial in $\mathbb{Q}[X]$. Then for each conjugacy class $C \subset \operatorname{Gal}\left(K_{\ell} / \mathbb{Q}\right)$,

$$
\operatorname{Frob}_{p} \in C \Leftrightarrow \Gamma_{C}\left(\operatorname{Tr}_{\frac{\mathbb{F}_{p}[x]}{P(x)} / \mathbb{F}_{p}}\left(h(x) x^{p}\right)\right) \equiv 0 \bmod p,
$$

where the polynomial $\Gamma_{C}(X)$ is given by

$$
\Gamma_{C}(X)=\prod_{\sigma \in C}\left(X-\sum_{i=1}^{\ell^{2}-1} h\left(a_{i}\right) \sigma\left(a_{i}\right)\right) .
$$

Our strategy for computing $\Gamma_{C}(X)$ is using Hensel lifting.

Proposition 4.6. Let $p$ be a prime such that the extension degree $d_{p}$ is in $O(1)$. Let us be given the polynomial $P(X)$ as in Section 4.2 and the Ramanujan subspace $V_{\ell} \bmod p$. Then for any conjugacy class $C \subset \operatorname{Gal}\left(K_{\ell} / \mathbb{Q}\right)$, the polynomial $\Gamma_{C}(X) \in$ $\mathbb{Q}[X]$ can be computed in $O\left(\ell^{6+\delta+\epsilon}\right)$ bit operations.

Proof. Set $\mathbb{F}_{q}:=\mathbb{F}_{p^{d_{p}}}$. We can lift each root $\tilde{\iota}(x) \in \mathbb{F}_{q}$ of $\tilde{P}(X) \in \mathbb{F}_{p}[X]$ to the root $\iota(x) \in \mathbb{Q}_{q}$ of $P(X) \in \mathbb{Q}_{p}[X]$ by Hensel's lemma, where $\mathbb{Q}_{q}$ is the unramified extension of $\mathbb{Q}_{p}$ with extension degree $d_{p}$. Since the heights of coefficients of $\Gamma_{C}(X)$ are bounded above by $N:=|C|(1+\operatorname{deg} h) \ell^{\delta}$, to recover $\Gamma_{C}(X)$ it suffices to lift each $\tilde{\iota}(x)$ to $\iota(x)$ with precision $N$. The complexity of a single lifting is about $\mathrm{O}\left(\left(|C|\left(d_{p} N\right)\right)^{1+\epsilon}\right)$ bit operations; see [2. The length of the conjugacy class $C$ is in $\mathrm{O}\left(\ell^{2}\right)$, the extension degree $d_{p}$ is in $\mathrm{O}(1)$ and $h(X)$ can be chosen to be a polynomial with small degree (e.g. $\operatorname{deg} h(X)=2$ ) shown in [12, so the complexity of lifting a single root is in $\mathrm{O}\left(\ell^{4+\delta+\epsilon}\right)$. There are $\ell^{2}-1$ roots need to be lifted, so the complexity of computing $\Gamma_{C}(X)$ is $\mathrm{O}\left(\ell^{6+\delta+\epsilon}\right)$.

Remark 4.7. The Galois group $\operatorname{Gal}\left(K_{\ell} / \mathbb{Q}\right)$ is a subgroup of $\mathrm{GL}_{2}\left(\mathbb{F}_{\ell}\right)$, and the action of $\operatorname{Gal}\left(K_{\ell} / \mathbb{Q}\right)$ on the roots $a_{i}, 1 \leq i \leq \ell^{2}-1$, can be computed from the action of $\mathrm{GL}_{2}\left(\mathbb{F}_{\ell}\right)$ on $V_{\ell} \bmod p$. In practice, we would like to choose the prime $p$ with large size and small extension degree $d_{p}$ (e.g. $\left.d_{p}=2\right)$ at the same time.

4.4. Complexity analysis. Now we can prove Theorem 1.1 and Corollary 1.2 As shown above, the complexity of computing $P(X)$ is $\mathrm{O}\left(\ell^{6+2 \omega+\delta+\epsilon}\right)$. There are $\ell^{2}-1$ conjugacy classes in $\mathrm{GL}_{2}\left(\mathbb{F}_{\ell}\right)$. So the complexity of computing $\Gamma_{C}(X)$ for all the conjugacy classes $C \subset \mathrm{GL}_{2}\left(\mathbb{F}_{\ell}\right)$ is $\mathrm{O}\left(\ell^{8+\delta+\epsilon}\right)$.

Let $p$ be a prime not equal to $\ell$. Denote the polynomial $P(X)$ as

$$
P(X)=X^{\ell^{2}-1}+c_{\ell^{2}-2} X^{\ell^{2}-2}+\ldots+c_{0} .
$$

The trace in (4.4) can be interpreted as a trace of a matrix

$$
\operatorname{Tr}_{\frac{\mathbb{F}_{p}[x]}{P(x)} / \mathbb{F}_{p}}\left(x^{d}\right)=\operatorname{Tr}\left(\begin{array}{cccc}
0 & & & -c_{0} \\
1 & & & -c_{1} \\
& \ddots & & \vdots \\
& & 1 & -c_{\ell^{2}-1}
\end{array}\right)^{d} \bmod p .
$$

Using the Coppersmith-Winograd algorithm, the complexity of multiplying two $\ell^{2} \times$ $\ell^{2}$ matrices over $\mathbb{F}_{p}$ is in $\mathrm{O}\left(\ell^{4.752} \log ^{1+\epsilon} p\right)$ bit operations. So $t:=\operatorname{Tr}_{\frac{\mathbb{F}_{p}[x]}{P(x)} / \mathbb{F}_{p}}\left(h(x) x^{p}\right)$ can be computed in $\mathrm{O}\left(\ell^{4.752} \log ^{2+\epsilon} p\right)$. Given $t$, the complexity of checking whether 
$\Gamma_{C}(t) \bmod p$ is equal to zero for all the conjugacy classes $C$ is bounded above by $\mathrm{O}\left(\ell^{4} \log ^{1+\epsilon} p\right)$.

So, for any prime $p \neq \ell$, the complexity of computing $\tau(p) \bmod \ell$ consists of the complexity of computing $P(X), \Gamma_{C}(X), \operatorname{Tr}\left(h(x) x^{p}\right)$ and $\Gamma_{C}\left(\operatorname{Tr}\left(h(x) x^{p}\right)\right)$ for all conjugacy classes $C \subset \mathrm{GL}_{2}\left(\mathbb{F}_{\ell}\right)$, which sums up to

$$
\mathrm{O}\left(\ell^{6+2 \omega+\delta+\epsilon}\right)+\mathrm{O}\left(\ell^{4.752} \log ^{2+\epsilon} p\right)+\mathrm{O}\left(\ell^{4} \log ^{1+\epsilon} p\right) .
$$

For prime $p$, we have $\tau(p) \in \mathrm{O}\left(p^{6}\right)$. Therefore it suffices to recover $\tau(p)$ from $\tau(p) \bmod \ell$ with $\ell \leq L$, where $L$ is in $\mathrm{O}(\log p)$. So the total complexity of computing $\tau(p)$ is about

$$
\sum_{\ell<L, \text { prime }} \mathrm{O}\left(\ell^{6+2 \omega+\delta+\epsilon}\right)+\mathrm{O}\left(\ell^{4.752} \log ^{2+\epsilon} p\right)+\mathrm{O}\left(\ell^{4} \log ^{1+\epsilon} p\right)=\mathrm{O}\left(\log ^{7+2 \omega+\delta+\epsilon} p\right) .
$$

Remark 4.8. If the constant $\omega$ reaches 2.376 , the complexity of the algorithm is $\mathrm{O}\left(\log ^{11.752+\delta+\epsilon} p\right)$. Moreover, if $\delta$ is bounded above by 3 , the complexity is $\mathrm{O}\left(\log ^{14.752+\epsilon} p\right)$.

\section{ImPLEMENTATION AND RESUlts}

The algorithm has been implemented in MAGMA. One big advantage of the algorithm is that it is rather straightforward to implement, where the major work is dealing with the action of Hecke operators on divisors of the function field. The following computation was done on a personal computer AMD FX(tm)-6200 SixCore Processor $3.8 \mathrm{GHz}$.

Let $Q_{\ell}(x)$ be the polynomial corresponding to the projective representation, defined as

$$
Q_{\ell}(X):=\prod_{L \in \mathbb{P}\left(V_{\ell}\right)}\left(X-\sum_{\alpha \in L \backslash O} \iota(\alpha)\right)
$$

which can be used to check whether $\tau(p) \equiv 0 \bmod \ell$. More precisely, we have the following lemma; see [5].

Lemma 5.1. Let $Q_{\ell}(X)$ be the polynomial defined as above and $p \nmid \operatorname{Disc}\left(Q_{\ell}(X)\right)$ a prime. Then $\tau(p) \equiv 0 \bmod \ell$ if and only if $Q_{\ell}(X) \bmod p$ has an irreducible factor of degree 2 over $\mathbb{F}_{p}$.

Example 5.2. $\ell=13$.

To recover $Q_{13}(X)$, it suffices to take a good prime set as

$\{19,23,29,43,53,61,67,71,79,83,89,109,127,149,157,163,179,193$, 
with a total of 24 primes, whose product is a 52-digit number. We have

$$
\begin{aligned}
2535853 \cdot Q_{13}(X) & =2535853 X^{14}+760835865 X^{13}+96570870461 X^{12} \\
& +7083218145770 X^{11}+341554192651282 X^{10} \\
& +11596551892957577 X^{9}+288394789072144586 X^{8} \\
& +5369247990154339694 X^{7}+75509842125272520446 X^{6} \\
& +800346109631330635243 X^{5}+6303044886777591079517 X^{4} \\
& +35793920471135235999031 X^{3}+138667955645963961606844 X^{2} \\
& +328650624808255716476451 X+361128579432826593902125 .
\end{aligned}
$$

The computation took several minutes.

The product of the primes needed to recover $P_{13}(X)$ is about 130 digits and the computation took nearly one hour.

In order to recover the polynomials $\Gamma_{C}(X)$ for $C \subset \mathrm{GL}_{2}\left(\mathbb{F}_{13}\right)$, we first choose a good prime $p=34939$ with $d_{p}=2$, and then compute the roots of $P_{13}(X) \bmod p$ by computing $V_{\ell} \bmod p$. Notice that all of the roots are in $\mathbb{F}_{p^{2}}$. Using the Hensel

\begin{tabular}{|c|c|c|c|c|}
\hline \multirow{2}{*}{ level } & $Q_{\ell}(X)$ & $P_{\ell}(X)$ & \multirow{2}{*}{$\begin{array}{c}\Gamma_{C}(X) \\
\text { time }\end{array}$} & \multirow{2}{*}{ Frob $_{p}$} \\
\hline & good primes time & good primes time & & \\
\hline 13 & 52 digits few minutes & 130 digits few minutes & few hours & {$\left[\begin{array}{cc}10 & 0 \\
0 & 7\end{array}\right]$} \\
\hline 17 & 467 digits few hours & 740 digits few hours & one day & {$\left[\begin{array}{cc}15 & 1 \\
0 & 15\end{array}\right]$} \\
\hline 19 & 832 digits few days & 1681 digits few days & few days & {$\left[\begin{array}{cc}17 & 1 \\
0 & 17\end{array}\right]$} \\
\hline
\end{tabular}
lemma, we lift each root to the $p$-adic field $\mathbb{Q}_{p^{2}}$ with a precision of nearly 5000 digits and then reconstruct $\Gamma_{C}(X)$ by the formula (4.5). The computation took several hours.

We summarize the results in the following table, where the matrices in the last column are the representatives of the conjugacy classes where the Frobenius endomorphism Frob $p$ lands inside and prime $p$ is set to be $10^{1000}+1357$.

Using a plane model for $X_{H}(31)$, we also finished the level 31 case. It took several days to recover the polynomial $Q_{31}(X)$. The coefficients of $Q_{31}(X)$ are very large, where the largest one reaches 2426 digits. Similar to the $\ell \in\{13,17,19\}$ cases, $Q_{31}(X)$ can be reduced to a polynomial with small coefficients. One of the reduced polynomials is

$$
\begin{aligned}
f_{31}= & X^{32}-4 X^{31}-155 X^{28}+713 X^{27}-2480 X^{26}+9300 X^{25}-5921 X^{24} \\
& +24707 X^{23}+127410 X^{22}-646195 X^{21}+747906 X^{20}-7527575 X^{19} \\
& +4369791 X^{18}-28954961 X^{17}-40645681 X^{16}+66421685 X^{15} \\
& -448568729 X^{14}+751001257 X^{13}-1820871490 X^{12}+2531110165 X^{11} \\
& -4120267319 X^{10}+4554764528 X^{9}-5462615927 X^{8}+4607500922 X^{7} \\
& -4062352344 X^{6}+2380573824 X^{5}-1492309000 X^{4}+521018178 X^{3} \\
& -201167463 X^{2}+20505628 X-1261963 .
\end{aligned}
$$


We follow the strategy proposed by Bosman (see [5], Chapter 3 or 13 Chapter 7 for the details) to prove that $f_{31}$ is the polynomial corresponding to the mod-31 projective representation associated to $\Delta(q)$. Using MAGMA, we have that the Galois group of the polynomial $f_{31}$ is isomorphic to $\mathrm{PGL}_{2}\left(\mathbb{F}_{31}\right)$ and the discriminant of the maximal order $\mathcal{O}_{K}$ of $K=\mathbb{Q}[X] /\left(f_{31}\right)$ is equal to $-31^{41}$. Let $\tilde{\rho}: \operatorname{Gal}(\overline{\mathbb{Q}} / \mathbb{Q}) \rightarrow$ $\mathrm{PGL}_{2}\left(\mathbb{F}_{31}\right)$ be a representation arising from $\operatorname{Gal}\left(f_{31}\right) \cong \mathrm{PGL}_{2}\left(\mathbb{F}_{31}\right)$. An important theorem of Tate on lifting (see 28 for the proof) and a theorem of Moon and Taguchi [26] show that there exists a representation $\rho: \operatorname{Gal}(\overline{\mathbb{Q}} / \mathbb{Q}) \rightarrow \mathrm{GL}_{2}\left(\mathbb{F}_{31}\right)$ lifting $\tilde{\rho}$ with weight 12 and level one. Now we use Serre's conjecture (the level one case proved by Khare and Wintenberger [21]) and the fact that $\Delta(q)$ is the unique newform in $S_{12}\left(\mathrm{SL}_{2}(\mathbb{Z})\right)$ to show $\rho \cong \rho_{31}$, where $\rho_{31}$ is the mod-31 representation associated to $\Delta(q)$.

An easy calculation shows that the first few primes satisfying Serre's criteria as well as $\tau(p) \equiv 0 \bmod 11 \cdot 13 \cdot 17 \cdot 19 \cdot 31$ are

$$
\begin{gathered}
982149821766199295999,3748991773540147199999, \\
3825907566871689215999,3903375187595059199999 .
\end{gathered}
$$

So we proved Theorem 1.4

From the table above, we have $\tau\left(10^{1000}+1357\right) \equiv 15 \bmod 19$. So the missing sign in the table of [13] is found. Moreover, we have

$$
\tau\left(10^{1000}+1357\right) \equiv \pm 18 \bmod 31 .
$$

The Magma code of our algorithm can be downloaded from the web at the address

http://faculty.math.tsinghua.edu.cn/ lsyin/publication.htm.

\section{ACKNOWLEDGMENTS}

Our interest in computing coefficients of modular forms was motivated by the wonderful courses given by Bas Edixhoven and Jean-Marc Couveignes at Tsinghua University. Many thanks to them for their encouragement. The first author wishes to thank Jean-Marc Couveignes for his continuous assistance and many helpful suggestions. Thanks to Ye Tian for his helpful comments. Many thanks to Maarten Derickx for fruitful discussions, helpful comments and suggestions. Thanks to Mark van Hoeij for providing us with a plane model for $X_{H}(31)$ and helping us reduce the polynomial $Q_{31}(X)$; these made the computation of level 31 a reality.

\section{REFERENCES}

[1] Avner Ash and Glenn Stevens, Modular forms in characteristic $l$ and special values of their L-functions, Duke Math. J. 53 (1986), no. 3, 849-868, DOI 10.1215/S0012-7094-86-05346-9. MR 860675 (88h:11036)

[2] Henri Cohen, Gerhard Frey, Roberto Avanzi, Christophe Doche, Tanja Lange, Kim Nguyen, and Frederik Vercauteren (eds.), Handbook of Elliptic and Hyperelliptic Curve Cryptography, Discrete Mathematics and its Applications (Boca Raton), Chapman \& Hall/CRC, Boca Raton, FL, 2006. MR2162716 (2007f:14020)

[3] Houria Baaziz, Equations for the modular curve $X_{1}(N)$ and models of elliptic curves with torsion points, Math. Comp. 79 (2010), no. 272, 2371-2386, DOI 10.1090/S0025-5718-1002332-X. MR2684370 (2011i:11056)

[4] Wieb Bosma, John Cannon, and Catherine Playoust, The Magma algebra system. I. The user language, J. Symbolic Comput. 24 (1997), no. 3-4, 235-265, DOI 10.1006/jsco.1996.0125. Computational algebra and number theory (London, 1993). MR.1484478 
[5] J. Bosman, Explicit computations with modular Galois representations, Ph.D. thesis, Universiteit Leiden, December 2008. Available on https://openaccess.leidenuniv.nl/

[6] A. Bostan, F. Morain, B. Salvy, and É. Schost, Fast algorithms for computing isogenies between elliptic curves, Math. Comp. 77 (2008), no. 263, 1755-1778, DOI 10.1090/S00255718-08-02066-8. MR2398793 (2009k:11207)

[7] P.J. Bruin, Modular curves, Arakelov theory, algorithmic applications, Ph.D. thesis, Universiteit Leiden, 2010. Available on http://www.math.leidenuniv.nl/en/theses/PhD/

[8] C. Citro and A. Ghitza, Computing level one Hecke eigensystems $(\bmod p)$, LMS J. Comput. Math. 16 (2013), 246-270. MR3104940

[9] J.-M. Couveignes, Linearizing torsion classes in the Picard group of algebraic curves over finite fields, J. Algebra 321 (2009), no. 8, 2085-2118, DOI 10.1016/j.jalgebra.2008.09.032. MR2501511 (2010e:14019)

[10] M. Derickx, Torsion points on elliptic curves and gonalities of modular curves, Master thesis, Universiteit Leiden, 2012. Available on http://www.mderickx.nl/

[11] Fred Diamond and Jerry Shurman, A First Course in Modular Forms, Graduate Texts in Mathematics, vol. 228, Springer-Verlag, New York, 2005. MR2112196 (2006f:11045)

[12] Tim Dokchitser and Vladimir Dokchitser, Identifying Frobenius elements in Galois groups, Algebra Number Theory 7 (2013), no. 6, 1325-1352, DOI 10.2140/ant.2013.7.1325. MR3107565

[13] Computational Aspects of Modular Forms and Galois Representations, Annals of Mathematics Studies, vol. 176, Princeton University Press, Princeton, NJ, 2011. How one can compute in polynomial time the value of Ramanujan's tau at a prime; Edited by Bas Edixhoven and Jean-Marc Couveignes. MR2849700

[14] Benedict H. Gross, A tameness criterion for Galois representations associated to modular forms $(\bmod p)$, Duke Math. J. 61 (1990), no. 2, 445-517, DOI 10.1215/S0012-7094-90-061198. MR1074305 (91i:11060)

[15] E. Grosswald, On Burgess' bound for primitive roots modulo primes and an application to $\Gamma(p)$, Amer. J. Math. 103 (1981), no. 6, 1171-1183, DOI 10.2307/2374229. MR636957 $(82 \mathrm{k}: 10059)$

[16] F. Hess, Computing Riemann-Roch spaces in algebraic function fields and related topics, J. Symbolic Comput. 33 (2002), no. 4, 425-445, DOI 10.1006/jsco.2001.0513. MR1890579 (2003j:14032)

[17] M. van Hoeij, Low Degree Places on the Modular Curve $X_{1}(N)$, http://arxiv.org/abs/ $1202.4355 \mathrm{v} 2$

[18] Ming-Deh Huang and Doug Ierardi, Efficient algorithms for the Riemann-Roch problem and for addition in the Jacobian of a curve, J. Symbolic Comput. 18 (1994), no. 6, 519-539, DOI 10.1006/jsco.1994.1063. MR1334660 (96h:14077)

[19] Naomi Jochnowitz, A study of the local components of the Hecke algebra mod l, Trans. Amer. Math. Soc. 270 (1982), no. 1, 253-267, DOI 10.2307/1999771. MR642340 (83e:10033a)

[20] Naomi Jochnowitz, Congruences between systems of eigenvalues of modular forms, Trans. Amer. Math. Soc. 270 (1982), no. 1, 269-285, DOI 10.2307/1999772. MR642341(83e:10033b)

[21] Chandrashekhar Khare, Serre's modularity conjecture: the level one case, Duke Math. J. 134 (2006), no. 3, 557-589, DOI 10.1215/S0012-7094-06-13434-8. MR2254626 (2007e:11060)

[22] Kamal Khuri-Makdisi, Asymptotically fast group operations on Jacobians of general curves, Math. Comp. 76 (2007), no. 260, 2213-2239 (electronic), DOI 10.1090/S0025-5718-07-019898. MR2336292(2009a:14072)

[23] Chang Heon Kim and Ja Kyung Koo, Generators of function fields of the modular curves $X_{1}(5)$ and $X_{1}(6)$, Math. Comp. 79 (2010), no. 270, 1047-1066, DOI 10.1090/S0025-5718-0902303-5. MR2600555 (2011d:11091)

[24] J. C. Lagarias and A. M. Odlyzko, Effective versions of the Chebotarev density theorem, Algebraic number fields: $L$-functions and Galois properties (Proc. Sympos., Univ. Durham, Durham, 1975), Academic Press, London, 1977, pp. 409-464. MR0447191 (56 \#5506)

[25] N. Mascot, Computing modular Galois representations, Rend. Circ. Mat. Palermo (2) 62 (2013), no. 3, 451-476. MR.3118315

[26] Hyunsuk Moon and Yuichiro Taguchi, Refinement of Tate's discriminant bound and nonexistence theorems for mod p Galois representations, Doc. Math. Extra Vol. (2003), 641-654 (electronic). Kazuya Kato's fiftieth birthday. MR2046611(2005a:11069) 
[27] Jean-Pierre Serre, Quelques applications du théorème de densité de Chebotarev (French), Inst. Hautes Études Sci. Publ. Math. 54 (1981), 323-401. MR644559 (83k:12011)

[28] J.-P. Serre, Modular forms of weight one and Galois representations, Algebraic number fields: $L$-functions and Galois properties (Proc. Sympos., Univ. Durham, Durham, 1975), Academic Press, London, 1977, pp. 193-268. MR0450201 (56 \#8497)

[29] Jacob Sturm, On the congruence of modular forms, Number theory (New York, 1984), Lecture Notes in Math., vol. 1240, Springer, Berlin, 1987, pp. 275-280, DOI 10.1007/BFb0072985. MR894516 (88h:11031)

[30] Andrew V. Sutherland, Constructing elliptic curves over finite fields with prescribed torsion, Math. Comp. 81 (2012), no. 278, 1131-1147, DOI 10.1090/S0025-5718-2011-02538-X. MR2869053 (2012m:11079)

[31] Emil J. Volcheck, Computing in the Jacobian of a plane algebraic curve, Algorithmic number theory (Ithaca, NY, 1994), Lecture Notes in Comput. Sci., vol. 877, Springer, Berlin, 1994, pp. 221-233, DOI 10.1007/3-540-58691-1_60. MR.1322725 (96a:14033)

Department of Mathematical Science, Tsinghua University, Beijing 100084, People's Republic of China

E-mail address: cengjx09@mails.tsinghua.edu.cn

Department of Mathematical Science, Tsinghua University, Beijing 100084, People's Republic of ChinA

E-mail address: 1syin@math.tsinghua.edu.cn 